\title{
A Scaled Difference Chi-square Test Statistic for Moment Structure Analysis *
}

\author{
Albert Satorra \\ Universitat Pompeu Fabra \\ and \\ Peter M. Bentler \\ University of California, Los Angeles
}

August 3, 1999

* Research supported by the Spanish DGES grant PB96-0300, and USPHS grants DA00017 and DA01070. 


\begin{abstract}
A family of scaling corrections aimed to improve the chi-square approximation of goodness-of-fit test statistics in small samplesTlarge modelsTand nonnormal data was proposed in Satorra and Bentler (1994). For structural equations modelsTSatorra-Bentler's (SB) scaling corrections are available in standard computer software. Often Thowever Tthe interest is not on the overall fit of a model Cbut on a test of the restrictions that a null model say $\mathcal{M}_{0}$ implies on a less restricted one $\mathcal{M}_{1}$. If $T_{0}$ and $T_{1}$ denote the goodness-of-fit test statistics associated to $\mathcal{M}_{0}$ and $\mathcal{M}_{1}$ Trespectively ce $T_{d}=T_{0}-T_{1}$ is used as a chi-square test statistic with degrees of freedom equal to the difference on the number of independent parameters estimated under the models $\mathcal{M}_{0}$ and $\mathcal{M}_{1}$. As in the case of the goodness-of-fit test is of interest to scale the statistic $T_{d}$ in order to improve its chi-square appro-

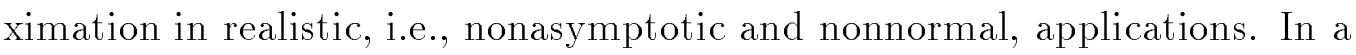
recent paper $\Gamma$ Satorra (1999) shows that the difference between two SatorraBentler scaled test statistics for overall model fit does not yield the correct SB scaled difference test statistic. Satorra developed an expression that permits scaling the difference test statistic limitations $\Gamma$ since it requires heavy computations that are not available in standard computer software. The purpose of the present paper is to provide an easy way to compute the scaled difference chi-square statistic from the scaled goodness-of-fit test statistics of models $\mathcal{M}_{0}$ and $\mathcal{M}_{1}$. A Monte Carlo study is provided to illustrate the performance of the competing statistics.
\end{abstract}

Keywords: Moment-structures Tgoodness-of-fit test Tchi-square difference test statistic $\Gamma$ chi-square distribution $\Gamma$ non-normality 


\section{Introduction}

Moment structure analysis is widely used in behaviouralTsocial and economic studies to analyse structural relations between variables $\Gamma$ some of which may

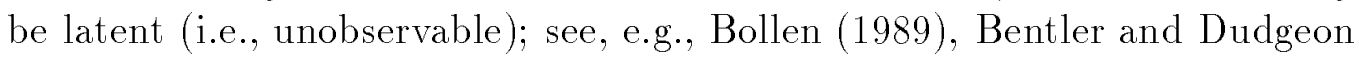
(1996) TYuan and Bentler (1997) Tand references therein. Commercial computer programs to carry out such analysis $\Gamma$ for a general class of structural

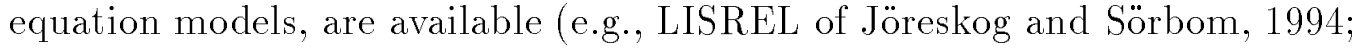
EQS of BentlerГ1995). In multi-sample analysis $\Gamma$ data from several samples are combined into one analysis $\Gamma$ making it possibleTamong other features $\Gamma$ to test for across-group invariance of specific model parameters. Statistics that are central in moment structure analysis are the overall goodness-of-fit test of the model and tests of restrictions on parameters.

Asymptotic distribution-free (ADF) methods which do not require distributional assumptions on the observable variables have been developed (BrowneГ1984). The ADF methods ThoweverTinvolve fourth-order sample moments Thus they may lack robustness to small and medium-sized samples. In the case of non-normal data Tan alternative to the ADF approach is to use a normal-theory estimation method in conjunction with asymptotic robust standard errors and test statistics (see Satorra 1992). Asymptotic robust test statistics Thowever may still lack robustness to small and medium-sized samples. As an alternative to asymptotically robust test statistics $\Gamma$ Satorra and Bentler (1994; Satorra and BentlerГ1988aIB) developed a family of corrected normal-theory test statistics which are easy to implement in practice and which have been shown to outperform the asymptotic robust test statistics in small and medium-sized samples (e.g. TChou Bentler and Sa-

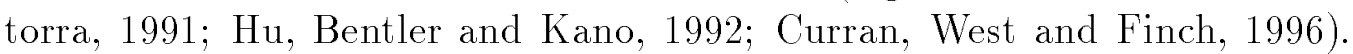
Bentler and Yuan (1999) provide a recent comparison of alternative test methods for small samples. Extension of Satorra-Bentler (SB)'s corrections to goodness-of-fit test statistics in the case of the analysis of augmented moment structures $\Gamma$ multi-samples and categorical data $\Gamma$ have been discussed respectively by Satorra (1992) and Muthén (1993).

Although SB corrections have been available for some timeTformal derivations of SB corrections to the case of nested model comparisons have not been available. The obvious and generally accepted approach of computing separate SB-corrected test statistics for each of two nested models Tand then computing the difference between them (e.g. $\mathrm{B}$ Byrne and CampbellГ1999) $\Gamma$ 
turns out to be an incorrect way to obtain a scaled SB difference test statistic. The difference could be even be negative which is an improper value for a chi-square variate. In a recent paperTSatorra (1999) gives specific formulae for extension of SB corrections to score (Lagrange multiplier) Tdifference and Wald test statistics. He showed that the difference between two SB-scaled test statistics does not necessarily correspond to the scaled chi-square difference test statistic. The purpose of the present paper is to provide a simple expression that allows a researcher to correctly compute the SB difference test statistic when the SB-scaled chi-square goodness of fit tests for the corresponding two nested models are available. The formula is simple to use and provides an alternative scaled test for evaluating a specific set of restrictions.

The paper is structured as follows. In Section 2 we describe goodness-offit tests in weighted least squares analysisTand the corresponding SB scaling corrections. In Section 3 we describe the proposed procedure for computing the SB scaled difference test statistic. Section 4 concludes with an illustration.

\section{Goodness-of-fit tests}

Let $\sigma$ and $s$ be $p$-dimensional vectors of population and sample moments respectively $\Gamma$ where $s$ tends in probability to $\sigma$ as sample size $n \rightarrow+\infty$. Let $\sqrt{n} s$ be asymptotically normally distributed with a finite asymptotic variance matrix $\Gamma(p \times p)$. Consider the model $\mathcal{M}_{0}: \sigma=\sigma(\theta)$ for the moment vector $\theta \Gamma$ where $\sigma($.$) is a twice-continuously differentiable vector-valued function of \theta \Gamma$ a $q$-dimensional parameter vector. Consider a WLS estimator $\hat{\theta}$ of $\theta$ defined as the minimizer of

$$
F_{V}(\theta):=(s-\sigma)^{\prime} \hat{V}(s-\sigma)
$$

over the parameter space $\Gamma$ where $\hat{V}(p \times p) \Gamma$ converges in probability to $V \Gamma$ a positive definite matrix. A typical test statistic used for testing the goodness-of fit-of the model $\mathcal{M}_{0}$ is $T_{0}:=n F_{V}(s, \hat{\sigma})$ Twhere $\hat{\sigma}:=\sigma(\hat{\theta})$. It is widely known that $\Gamma$ when the model $\mathcal{M}_{0}$ holds and $V$ satisfies the asymptotic optimality (AO) condition of $V=\Gamma^{-1} \Gamma$ then $T_{0}$ is asymptotically chi-square distributed with degrees of freedom $(\mathrm{df}) r_{0}=p-q$. In practiceГhowever $\Gamma A O$ may not hold Tand concern on the quality of the chi-square approximation do arise. For general types of distributionsTi.e. Twhen AO does not necessarily hold $\Gamma T_{0}$ is asymptotically distributed as a mixture of chi-square distributions 
of 1 degree of freedom (df) (see Satorra and BentlerГ1986); that is

$$
T_{0} \stackrel{L}{\rightarrow} \sum_{j=1}^{r} \alpha_{j} \chi_{j}^{2},
$$

as $n \rightarrow \infty \Gamma$ where the $\chi_{j}^{2}$ are independent chi-square variables of $1 \mathrm{df} \Gamma$ and the $\alpha_{j}$ are the non-null eigenvalues of the matrix $U_{0} \Gamma \Gamma$ with

$$
U_{0}:=V-V \Delta\left(\Delta^{\prime} V \Delta\right)^{-1} \Delta^{\prime} V
$$

and $\Delta:=\left(\partial / \partial \theta^{\prime}\right) \sigma(\theta)$. When $A O$ holds $\Gamma$ then of course the $\alpha_{j}$ 's are equal to 1 and the asymptotic exact chi-square distribution applies. In the context of structural models and for general types of distributions TSatorra and Bentler (1994; Satorra and Bentler $\Gamma 1988 \mathrm{aIb})$ proposed replacing $T$ by the scaled statistic

$$
\bar{T}=T / \hat{c},
$$

where $\hat{c}$ denotes a consistent estimator of

$$
c:=\frac{1}{r} \operatorname{tr} U_{0} \Gamma=\frac{1}{r} \operatorname{tr}\{V \Gamma\}-\frac{1}{r} \operatorname{tr}\left\{\left(\Delta^{\prime} V \Delta\right)^{-1} \Delta^{\prime} V \Gamma V \Delta\right\} .
$$

Note that the SB scaled test statistic has the same mean as the corresponding $\chi_{r}^{2}$ variate. The SB scaled goodness-of-fit test has been shown to outperform alternative test statistics in a variety of models and non-normal distributions

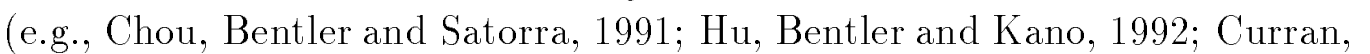
West and Finch 1996 ). Of course when asymptotic optimality holds $\Gamma$ this statistic will have the same asymptotic distribution as the unscaled statistic $T_{0}$. Note that a consistent estimator $\hat{\Gamma}$ of $\Gamma$ under general distribution conditions is required to compute the scaling factor $\hat{c}$. In structural equation models $\Gamma$ a consistent estimator of $\Gamma$ is readily available from the raw data (e.g. SSatorra and BentlerГ1994). A goodness-of-fit statistic which can be used given any estimation method ${ }^{2}$ is given by

$$
T^{\star}=n(s-\hat{\sigma})^{\prime}\left\{\hat{\Gamma}^{-1}-\hat{\Gamma}^{-1} \hat{\Delta}\left(\hat{\Delta}^{\prime} \hat{\Gamma}^{-1} \hat{\Delta}\right)^{-1} \hat{\Delta}^{\prime} \hat{\Gamma}^{-1}\right\}(s-\hat{\sigma}),
$$

When $\hat{\Gamma}$ is the (distribution-free) consistent estimator of $\Gamma$ in (16) below $\Gamma$ then $T^{\star}$ will be called the asymptotic robust goodness-of-fit test statistic $\Gamma$ since it is an asymptotic chi-square statistic regardless of the distribution of observable variables. In the context of single-sample covariance structure 
analysis $\mathrm{Tthis}$ statistic was first introduced by Browne (1984). Its performance was studied by Yuan and Bentler (1998) Twho found that very large samples are required to obtain acceptable performance in models with intermediate to large degrees of freedom.

\section{Testing a set of restrictions}

Consider now the case of testing a specific set of restrictions on the model. Consider a re-parameterezation of $\mathcal{M}_{0}$ as $\sigma=\sigma^{\star}(\delta)$ with $a(\delta)=a_{0} \Gamma$ where $\delta$ is a $(q+m)$-dimensional vector of parameters $\Gamma a_{0}$ is an $m \times 1$ vector of constants $\Gamma$ and $\sigma^{\star}($.$) and a($.$) are twice-continuously differentiable vector-$ valued functions of $\delta \in \Theta_{1} \Gamma$ a compact subset of $R^{q+m}$. Our interest now is in the test of the null hypothesis $H_{0}: a(\delta)=a_{0}$ against the alternative $H_{1}: a(\delta) \neq a_{0}$. Define the Jacobian matrices

$$
\Pi(p \times(q+m)):=\left(\partial / \partial \delta^{\prime}\right) \sigma^{\star}(\delta) \text { and } A(m \times(q+m)):=\left(\partial / \partial \delta^{\prime}\right) a(\delta),
$$

which we assume to be regular at the value of $\delta$ associated with $\theta_{0} \Gamma$ say $\delta_{0} \Gamma$ with $A$ of full row rank. Let $P((q+m) \times(q+m)):=\Pi^{\prime} V \Pi$ and denote by $\mathcal{M}_{1}$ the less restricted model $\sigma=\sigma^{\star}(\delta)$. The goodness-of-fit test statistic associated with $\mathcal{M}_{1}$ is thus $T_{1}=n F(s, \tilde{\sigma}) \Gamma$ where $\tilde{\sigma}$ is the fitted moment vector in model $\mathcal{M}_{1}$ Tnow with associated degrees of freedom $r_{1}=r_{0}-m$ and scaling factor $c_{1}$ given by

$$
c_{1}:=\frac{1}{r_{1}} \operatorname{tr} U_{1} \Gamma=\frac{1}{r_{1}} \operatorname{tr}\{V \Gamma\}-\frac{1}{r_{1}} \operatorname{tr}\left\{P^{-1} \Pi^{\prime} V \Gamma V \Pi\right\}
$$

where

$$
U_{1}:=V-V \Pi P^{-1} \Pi^{\prime} V .
$$

When both models $\mathcal{M}_{0}$ and $\mathcal{M}_{1}$ are fitted $\Gamma$ then we can test the restrictions $a(\theta)=a_{0}$ using the difference test statistic $T_{d}=T_{0}-T_{1}$ Twhere under the null hypothesis $\Gamma i t$ is intended that $T_{d}$ have a chi-square distributed with degrees of freedom $m=r_{0}-r_{1}$.

In order to improve the chi-square approximation in the case of large values of $m$ and moderate or small sample sizes $\Gamma$ we are interested in the SB scaled difference test statistic $\Gamma$ say $\bar{T}_{d}$. Extending his earlier work (Satorra $\Gamma$ 1989) ГSatorra (1999) recently provided formulae for computing such scaled 
statistics for the differenceГScore and Wald test statistics. From Satorra's formulae it becomes aparent that the SB scaled difference test statistic does not coincide with the difference between the two SB scaled goodness-of-fit test statistics that arise when fitting the two nested models; that is Tin general $\bar{T}_{d} \neq \bar{T}_{0}-\bar{T}_{1}$ Twhere by $\bar{T}_{0}$ and $\bar{T}_{1}$ we denote the SB scaled goodness-of-fit test statistics arising when fitting the models $\mathcal{M}_{0}$ and $\mathcal{M}_{1}$ respectively. In Satorra (1999) $\Gamma$ the SB scaled difference test statistic is defined as $\bar{T}_{d}=T_{d} / \hat{c}_{d}$ where $\hat{c}_{d}$ is a consistent estimator of

$$
c_{d}:=\frac{1}{m} \operatorname{tr} U_{d} \Gamma
$$

with

$$
U_{d}=V \Pi P^{-1} A^{\prime}\left(A P^{-1} A^{\prime}\right)^{-1} A P^{-1} \Pi^{\prime} V .
$$

A practical problem with this expression for the scaled difference test statistic is it requires computations that are outside the standard output of current structural equation modeling programs. FurthermoreTdifference tests are usually hand computed from different modeling runs. Here we will show how to combine the scaling corrections $c_{0}$ and $c_{1}$ associated to the two fitted models $\mathcal{M}_{0}$ and $\mathcal{M}_{1}$ in order to compute the scaling correction $c_{d}$ for the difference test statistic. It turns out that the computations are extremely simply and can be carried out using a hand calculator.

First we show that $U_{d}=U_{0}-U_{1}$. Note that the model $\mathcal{M}_{0}$ implies a specific function $\delta=\delta(\theta)$ Tthat by the implicit function theorem is continuous differentiable. Consider thus the matrix $H=\partial \delta(\theta) / \partial \theta^{\prime}$. ClearlyTit holds that $\Delta=\Pi H$ and $A H=0$ (recall that $A$ is a matrix $m \times(q+m)$ ) $\Gamma$ that is $\Gamma$ the matrix $A$ and $H$ are orthogonal complements. We have

$$
\begin{gathered}
U_{0}-U_{1}=V \Pi\left(\Pi^{\prime} V \Pi\right)^{-1} \Pi^{\prime} V-V \Pi H\left(H^{\prime} \Pi^{\prime} V \Pi H\right)^{-1} H^{\prime} \Pi^{\prime} V \\
=V \Pi\left\{P^{-1}-H\left(H^{\prime} P H\right)^{-1} H^{\prime}\right\} \Pi^{\prime} V
\end{gathered}
$$

since

$$
P^{-1}-H\left(H^{\prime} P H\right)^{-1} H^{\prime}=P^{-1} A^{\prime}\left(A P^{-1} A^{\prime}\right)^{-1} A P^{-1},
$$

as $A$ and $H$ are orthogonal complements (see RaoT1973Г p. 77). We thus have the basic result that

$$
U_{d}=U_{0}-U_{1}
$$


Now $\Gamma$ since $r_{0} c_{0}-r_{1} c_{1}=\operatorname{tr}\left(U_{0}-U_{1}\right) \Gamma=\operatorname{tr} U_{d} \Gamma=m c_{d}$, we obtain $c_{d}=$ $\left(r_{0} c_{0}-r_{1} c_{1}\right) / m$. This means that consistent estimation of $c_{d}$ is available from consistent estimates of the scaling corrections $c_{1}$ and $c_{1}$ associated with the null and alternative model respectively.

Thus the proposed practical procedure is as follows. When fitting models $\mathcal{M}_{0}$ and $\mathcal{M}_{1}$ Twe obtain the unscaled and scaled goodness-of-fit tests $\Gamma$ that is $T_{0}$ and $\bar{T}_{0}$ when fitting $\mathcal{M}_{0}$ Tand $T_{1}$ and $\bar{T}_{1}$ when fitting $\mathcal{M}_{1}$. Let $r_{0}$ and $r_{1}$ be the associated degrees of freedom of the goodness-of-fit test statistics. Then we compute the scaling corrections $\hat{c}_{0}=T_{0} / \bar{T}_{0}$ and $\hat{c}_{1}=T_{1} / \bar{T}_{1}$ T and the usual chi-square difference $T_{d}=T_{0}-T_{1}$. The SB scaled difference test can thus be computed as $\bar{T}_{d}=T / \hat{c}_{d}$, where

$$
\hat{c}_{d}=\left(r_{0} \hat{c}_{0}-r_{1} \hat{c}_{1}\right) / m \text {. }
$$

When the two scaling corrections are equalii.e. when $c_{0}=c_{1}=c$ then $c_{d}=c$ and thus $\bar{T}_{d}=\bar{T}_{0}-\bar{T}_{1}$. This is the case $\Gamma$ for example $\Gamma$ when $c_{0}=c_{1}=1 \Gamma$ i.e. Twhen both goodness-of-fit tests are asymptotically chi-square statistics. In general $\Gamma$ however $\Gamma c_{0} \neq c_{1}$ and then the difference between two SB scaled goodness of fit test statistics does not yield the SB scaled difference test statistic.

Note that the above procedure applies to a general modeling setting. The vector of parameters $\sigma$ to be modeled may contain various types of moments: means $\Gamma$ product-moments $\Gamma$ frequencies (proportions) Tand so forth. Thus $\Gamma$ the procedure applies to a variety of techniques $\Gamma$ such as factor analysis $\Gamma$ simultaneous equations for continuous variables $\Gamma \log$-linear multinomial parametric models $\Gamma$ etc.. It can easily be seen that the procedure applies also in the case where the matrix $\Gamma$ is singular $\Gamma$ and when the data is composed of various samples $\Gamma$ as in multi-sample analysis. The results apply also to other estimation methodsTe.g. Tpseudo ML estimation.

It is important to recognize that a competitor to the statistic $\bar{T}_{d}$ will be the difference between the robust goodness-of-fit test statistics associated with the models $\mathcal{M}_{0}$ and $\mathcal{M}_{1}$; that is $\Gamma$ an asymptotic chi-square test statistic for $H_{0}$ is just $T_{d}^{\star}:=T_{0}^{\star}-T_{1}^{\star} \Gamma$ where $T_{0}^{\star}$ and $T_{1}^{\star}$ are the goodness-of-fit test statistics associated to the models $\mathcal{M}_{0}$ and $\mathcal{M}_{1}$ respectively. In the next section Twe will illustrate using Monte Carlo simulation the small sample size performance of the competing test statistics for the above mentioned null hypothesis $H_{0}$. 


\section{Illustration}

In this section we ilustrate in a simple model context of a regression with errors in variables the performance in finite samples of three test statistics. We consider a regression equation

$$
y_{g i}^{\star}=\beta x_{g i}+v_{g i}, \quad i=1, \ldots, n_{g},
$$

where for case $i$ in group $g(g=1,2) \Gamma y_{g i}^{\star}$ and $x_{g i}$ are the values of the response and explanatory variables $\Gamma$ respectively $\Gamma v_{g i}$ is the value of the disturbance term $\Gamma$ and $\beta$ is the regression coefficient. The model assumes that $x_{g i}$ is unobservableTbut there are two observable variables $x_{1 g i}^{\star}$ and $x_{2 g i}^{\star}$ related to $x_{g i}$ by the following measurement-error equations

$$
x_{1 g i}^{\star}=x_{g i}+u_{1 g i}, \quad x_{2 g i}^{\star}=x_{g i}+u_{2 g i},
$$

where $u_{1 g i}$ and $u_{2 g i}$ are mutually independent and also independent of $v_{g i}$ and $x_{g i}$. It is assumed that the observations are independent and identically distributed within each group. Equations (8) and (9) with the associated assumptions yield an identified model (see Fuller (1987) for a comprehensive overview of measurement-error models in regression analysis). Inference is usually carried out in this type of model under the assumption that the observable variables are normally distributed. Write the model of (8) and (9) as

$$
z_{g i}=\Lambda \xi_{g i}, \quad i=1,2, \ldots, n,
$$

where

$$
z_{g i}:=\left(\begin{array}{c}
y_{g i}^{\star} \\
x_{1 g i}^{\star} \\
x_{2 g i}^{\star}
\end{array}\right), \quad \xi_{g i}:=\left(\begin{array}{c}
x_{g i} \\
v_{g i} \\
u_{1 g i} \\
u_{2 g i}
\end{array}\right)
$$

and

$$
\Lambda:=\left(\begin{array}{cccc}
\beta & 1 & 0 & 0 \\
1 & 0 & 1 & 0 \\
1 & 0 & 0 & 1
\end{array}\right)
$$

Define

$$
\Phi:=E \xi_{g i} \xi_{g i}^{\prime}=\left(\begin{array}{cccc}
\sigma_{x x} & 0 & 0 & 0 \\
0 & \sigma_{v v} & 0 & 0 \\
0 & 0 & \sigma_{u u} & 0 \\
0 & 0 & 0 & \sigma_{u u}
\end{array}\right)
$$


and the parameter vector $\theta:=\left(\sigma_{v v}, \sigma_{x x}, \sigma_{u u}, \beta\right)^{\prime}$. Under this set-up $\Gamma$ we obtain the moment structure

$$
\Sigma:=\Lambda \Phi \Lambda^{\prime}=\Sigma(\theta),
$$

where $\Sigma(.) \Gamma \Lambda($.$) and \Phi($.$) are (twice-continuously differentiable) matrix-$ valued functions of $\theta \Gamma$ as deduced from (11) $\Gamma(12)$ and (13). Note that the model restricts the variances of $u_{1}$ and $u_{2}$ by equality. This is a setting of two-sample data $\Gamma$ where the population and sample vectors $\sigma$ and $s$ are defined as $s=\left(\sigma_{1}^{\prime}, \sigma_{2}^{\prime}\right)^{\prime}$ and $s=\left(s_{1}^{\prime}, s_{2}^{\prime}\right)^{\prime} \Gamma$ where $\sigma_{g}^{\prime}=\operatorname{vec} S_{g}$ and $s_{g}^{\prime}=\operatorname{vec} S_{g} \Gamma$ with

$$
S_{g}:=\frac{1}{n_{g}} \sum_{i=1}^{n_{g}} z_{g i} z_{g i}^{\prime} .
$$

Here "vec" denotes the column-wise vectorisation operator (see Magnus and Neudecker $\Gamma 1999$ Гfor full details on this operator). We consider the estimation of the model using weighted least squares under the assumption of normality. That is The matrix $V$ (see above) has the form

$$
\hat{V}:=\text { block } \operatorname{diag}\left(\frac{n_{1}}{n} \hat{V}_{1}, \frac{n_{2}}{n} \hat{V}_{2}\right)
$$

and $\hat{V}_{g}=\frac{1}{2}\left(S_{g}{ }^{-1} \otimes S_{g}^{-1}\right) \Gamma g=1,2$. Clearly $\Gamma$ when there is independence across samples $\Gamma$ the asymptotic variance matrix of $\sqrt{n} s$ is of the form

$$
\Gamma=\text { block } \operatorname{diag}\left(\frac{n}{n_{1}} \Gamma_{1}, \frac{n}{n_{2}} \Gamma_{2}\right)
$$

where $\Gamma_{g}$ is the asymptotic variance of $\sqrt{n_{g}} s_{g} \Gamma g=1,2$. We further assume that the matrices $S_{g}$ and $\Sigma_{g}$ are positive definite $\Gamma$ and that $n_{g} / n \rightarrow f_{g}>0 \Gamma$ as $n \rightarrow+\infty(g=1,2)$; in this case $\Gamma$ a distribution-free consistent estimator of $\Gamma$ is

$$
\hat{\Gamma}:=\operatorname{block} \operatorname{diag}\left(\frac{n}{n_{1}} \hat{\Gamma}_{1}, \frac{n}{n_{2}} \hat{\Gamma}_{2}\right),
$$

where

$$
\hat{\Gamma}_{g}:=\frac{1}{n_{g}-1} \sum_{i=1}^{n_{g}}\left(d_{g i}-s_{g}\right)\left(d_{g i}-s_{g}\right)^{\prime},
$$

with $d_{g i}:=\operatorname{vec} z_{g i} z_{g i}^{\prime}$.

The Monte Carlo study generates two-sample data from the above model. Two models are fitted. Model $\mathcal{M}_{0}$ has the parameters restricted across groups $\Gamma$ and model $\mathcal{M}_{1}$ has parameters that are unrestricted across groups. 
For each of the estimated models $\Gamma$ we compute the goodness-of-fit test statistics $T_{0}$ and $T_{1} \Gamma$ the $\mathrm{SB}$ scaled statistics $\bar{T}_{0}$ and $\bar{T}_{1} \Gamma$ and the robust test statistics $T_{0}^{\star}$ and $T_{1}^{\star}$. To test the hypothesis of parameter invariance acrosssamples $\Gamma$ we consider the competing statistics $T_{d}=T_{0}-T_{1} \Gamma T_{d}^{\star}=T_{0}^{\star}-T_{1}^{\star} \Gamma$ $d \bar{T}=\bar{T}_{0}-\bar{T}_{1}$ and finally the proposed statistic $\bar{T}_{d}$. Note that only $T_{d}^{\star}$ is asymptotically an exact chi-square statistic. Our conjecture is that for nonnormal data 5 small samples and/or models with large degrees of freedom $\Gamma$ the statistic $\bar{T}_{d}$ will perform the best.

We obtained replications of the above statistics for various combinations of sample sizes Tranging from a small sample size to an intermediately large sample size. Results are reported in Table 1 and also in Figure 1 Twhere the empirical p-values of the various statistics are fitted against the theoretical ones corresponding to a uniform distribution. In all the replications we used $\theta_{0}=(1,1, .3,2)^{\prime}$. The distributions of $v$ and $x$ were independent conveniently scaled zero mean and unit variance chi-squared of $1 \mathrm{df}$ (i.e. Ta highly nonnormal distribution); the distribution of $u_{1}$ and $u_{2}$ were set to be normal $\Gamma$ mutually independent Tand independent of $v$ and $x$. The normal-theory GLS estimation method described in Section 2 was used. The restricted model imposed across-group invariance of model parameters. In each replicationTwe computed the statistics mentioned above $\mathrm{C}$ corresponding to the null hypothesis of invariance of cross-sample model parameters. ClearlyTin our Monte Carlo set-up Tthe null hypothesis holds trueTwith the null distribution of the statistics being chi-square with $m=4$ degrees of freedom. Note that in our Monte Carlo set-up Tsevere non-normality of random constituents of the model requires the use of robust and/or corrected versions of the difference test statistic. We note that the normal-theory chi-square goodness-of-fit $T_{1}$ of the unrestricted model (i.e. Tthe model that does not restrict parameters across groups) is an asymptotic chi-square statistic despite non-normality of the data (this follows from the asymptotic robustness theory for multi-samples; cf. $\Gamma$ SatorraГ1992). In contrast $\Gamma$ the normal-theory chi-square goodness of fit $T_{0}$ of the restricted model (i.e. Tthe model that imposes parameter invariance across-samples) is not necessarily an asymptotic chi-square statistic (since variances of non-normal constituents of the model are restricted by equality across-groups; cf.ГSatorraГ1992).

As shown in Table 1 Tin our specific model context Tin the smaller sample $\Gamma$ the SB scaled statistic $\Gamma \bar{T}_{d} \Gamma$ seems to outperform the alternative robust test statistic $T_{d}^{\star}$. As expected from theory $\mathrm{in}$ the case of the large sample $T_{d}^{\star}$ 
Table 1: Monte Carlo results:

empirical significance levels of test statistics

\begin{tabular}{|c|c|c|c|c|c|}
\hline nominal & I significance levels: & $1 \%$ & $\overline{5 \%}$ & $10 \%$ & $20 \%$ \\
\hline \multicolumn{6}{|c|}{$n_{1}=100$ and $n_{2}=120$} \\
\hline $\bar{T}_{d}$ & & 2.7 & 8.5 & 13.7 & 23.1 \\
\hline$T_{d}^{\star}$ & & 3.6 & 10.6 & 19.3 & 32.0 \\
\hline$T_{d}$ & & 24.8 & 39.5 & 50.1 & 62.1 \\
\hline$d \bar{T}$ & & 76.8 & 77.5 & 78.2 & 78.9 \\
\hline \multicolumn{6}{|c|}{$n_{1}=800$ and $n_{2}=900$} \\
\hline $\bar{T}_{d}$ & & 1.2 & 7.0 & 11.4 & 19.9 \\
\hline$T_{d}^{\star}$ & & .5 & 4.3 & 11.0 & 21.7 \\
\hline$T_{d}$ & & 29.8 & 43.1 & 52.5 & 62.8 \\
\hline$d \bar{T}$ & & 67.5 & 68.4 & 69.1 & 70 \\
\hline
\end{tabular}

outperforms the alternative test statistics. Especially interesting is that the statistic $d \bar{T}=\bar{T}_{0}-\bar{T}_{1}$ performs very badly indeed. That is $\Gamma$ doing the presumably natural thing $\Gamma$ simply computing the difference between two SB scaled chi-square statistics Tyields a very poorly performing test when evaluated by the chi-square distribution.

\section{References}

[1] BentlerГP. M. (1995). EQS structural equations program manual. EncinoГCA: Multivariate Software.

[2] BentlerГP. М.Tand DudgeonГP. (1996). Covariance structure analysis:

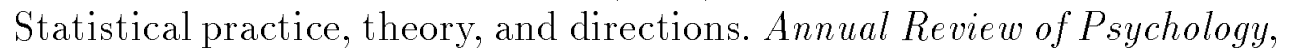
$47 \Gamma 541-570$.

[3] BentlerГP. М.Tand YuanГK. -H. (1999). Structural equation modeling with small samples: Test statistics. Multivariate Behavioral Research, $34 \Gamma 183-199$. 
[4] BollenTK. A. (1989). Structural equations with latent variables. New York: Wiley.

[5] BrowneTM. W. (1984). Asymptotically distribution-free methods for the analysis of covariance structures. British Journal of Mathematical and Statistical Psychology, 37Г62-83.

[6] ByrneГB. M. and CampbellГT. L. (1999). Cross-cultural comparisons and the presumption of equivalent measurement and theoretical structure: A look beneath the surface. Journal of Cross-cultural Psychology, $30 \Gamma 557-576$.

[7] ChouГC. -P.ГBentlerГP. М.Гand SatorraГА. (1991). Scaled test statistics and robust standard errors for non-normal data in covariance structure analysis: A Monte Carlo study. British Journal of Mathematical and Statistical Psychology, 44Г347-357.

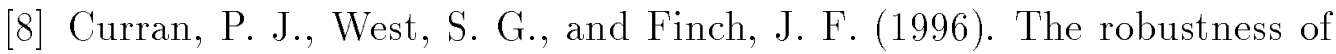
test statistics to nonnormality and specification error in confirmatory factor analysis. Psychological Methods, 1Г16-29.

[9] FullerTW. A. (1987). Measurement error models. New York: Wiley.

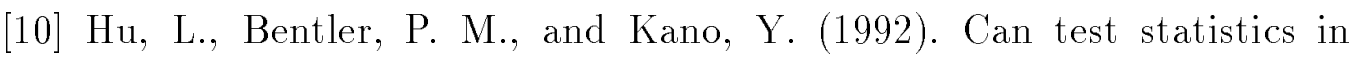
covariance structure analysis be trusted? Psychological Bulletin, $112 \Gamma$ $351-362$.

[11] Jöreskog ГK. Tand SörbomГD. (1994). LISREL 8 user's reference guide. MooresvilleГIN: Scientific Software.

[12] MagnusГJ.Tand NeudeckerГH. (1999). Matrix differential calculus with applications in statistics and econometrics. New York: Wiley.

[13] MuthénГB. (1993). Goodness of fit test with categorical and other nonnormal variables. In K. A. Bollen and J. S. Long (Eds.) T Testing structural equation models (pp. 205-234). Newbury Park: Sage.

[14] RaoГ C.R.Г(1973). Linear Statistical Inference and Its Applications $\Gamma$ 2nd. edit. New York: Wiley. 
[15] Satorra $\mathrm{A}$. (1989). Alternative test criteria in covariance structure analysis: A unified approach. Psychometrika, 54Г131-151.

[16] SatorraГA. (1992). Asymptotic robust inferences in the analysis of mean and covariance structures. Sociological Methodology, 22Г249-278.

[17] SatorraГA. (1999). Scaled and adjusted restricted tests in multi-sample analysis of moment structures. To appear in Innovations in Multivariate Statistical Multivariate Analysis: A Festschrift for Heinz Neudecker (R.D.H. HeijmansTD.S.G. PollockTand A. SatorraTedts. )TDordrecht: Kluwer Academic Publishers

[18] SatorraГ A.Tand BentlerГP. M. (1986). Some robustness properties of goodness of fit statistics in covariance structure analysis. $1986 \mathrm{ASA}$ Proceedings of the Business and Economic Statistics Section, 549-554Г AlexandriaTVA: American Statistical Association.

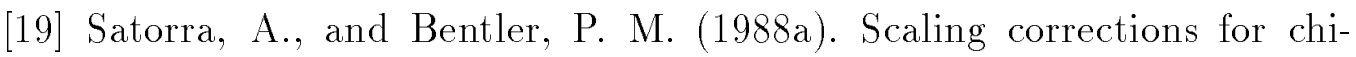
square statistics in covariance structure analysis. ASA 1988 Proceedings of the Business and Economic Statistics Section, 308-313Г AlexandriaГ VA: American Statistical Association.

[20] SatorraГА.Гand BentlerГP. M. (1988b). Scaling corrections for statistics in covariance structure analysis. UCLA Statistics Series \# 2, University of CaliforniaTLos Angeles.

[21] SatorraГА.Tand BentlerГP. M. (1994). Corrections to test statistics and standard errors in covariance structure analysis. In A. von Eye and C. C. Clogg (Eds.) TLatent variables analysis: Applications for developmental research (pp. 399-419). Thousand OaksГCA: Sage.

[22] YuanГK. -H.Гand BentlerГP. M. (1997). Mean and covariance structure analysis: Theoretical and practical improvements. Journal of The American Statistical Association, 92Г 767-774.

[23] YuanГK. -H. Tand BentlerTP. M. (1998). Normal theory based test statistics in structural equation modelling CBritish Journal of Mathematical and Statistical PsychologyГ51Г289-309. 